\title{
Evaluation of synergistic potential of plant growth promoting rhizobacteria with Rhizobium in mungbean (Vigna radiata $\mathbf{L}$.)
}

\author{
Satveer Kaur $^{1 *}$ and Veena Khanna ${ }^{2}$ \\ ${ }^{1}$ Department of Microbiology, Punjab Agricultural University, Ludhiana - 141001 (Punjab), INDIA \\ ${ }^{2}$ Department of Plant Breeding and Genetics, Punjab Agricultural University, Ludhiana - 141001 (Punjab), INDIA \\ *Corresponding author. E-mail: satveerbrar30@gmail.com \\ Received: September 3, 2015; Revised received: March 18, 2016; Accepted: June 4, 2016
}

\begin{abstract}
A pot experiment was conducted in glass house at PAU research farm, Ludhiana, Punjab, India to evaluate effect of co-inoculation of plant growth promoting rhizobacteria with Rhizobium on mungbean productivity. Coinoculation showed a significant increase at $5 \%$ in nodule number, nodule dry weight, shoot and root dry and fresh weight, plant length, no. of pods, chlorophyll and leghaemoglobin content, over Rhizobium alone. Application of PGPRs R-4, R-6, S-5, S-9 and S-11 along with Rhizobium further enhanced the grain yield over Rhizobium inoculation alone. Rizobacterial isolates R-6 and S-11 co-inoculated with Rhizobium showed better result than other isolates. These plant beneficial rhizobacteria may decrease the global dependence on various hazardous agricultural chemicals used in mungbean
\end{abstract}

Keywords: Co-inoculation, Mungbean, PGPR, Rhizobium

\section{INTRODUCTION}

Mungbean (Vigna radiata L. Wilczek) is an important pulse crop cultivated in Asia. India accounts about 1.94 million hectares area under mungbean cultivation of which Punjab occupied about 5.6 thousand hectare area with an average yield of $849 \mathrm{~kg} / \mathrm{ha}$ (Anonymous, 2013). It is short duration crop and have better storage ability. Moreover, it is drought resistant that can withstand adverse environmental conditions, and hence successfully be grown in rain fed areas (Anjum et al., 2006). Mungbean contains high amount of Vitamins $\mathrm{A}, \mathrm{B}, \mathrm{C}$ and niacin, and minerals such as potassium, phosphorus and calcium, which are necessary for human and commonly used as fodder and green manure. The scarcity of food containing high levels of protein, micronutrients and various vitamins sources is an increasing problem affecting millions of people in developing countries (Burchi et al., 2011). To improve the quality and yield of economically important legumes, particularly mungbean, farmers apply large quantities of chemical fertilizer, which has detrimental effects on the soil, such as the accumulation of toxic salts. Application of environmentally friendly and potentially cost effective microbial biofertilizer could be a better solution (Mia et al., 2010). PGPR is a group of bacteria that facilitate the hydrolysis of a wide range of compounds leading to higher crop yields and reduce chemical hazards to the environment. The seed bacterization with the appropriate rhizobia at sowing is a recommended practice, but in recent years seed bacterization with both PGPR and Rhizobium or their co-inoculation brought more balanced nutrition particularly under conditions of reduced nutrient inputs than single organism (Khanna and Sharma, 2011). The use of PGPR is steadily increasing in agriculture and offers an attractive alternative to chemical fertilizers, pesticides, and supplements (fuzzaman et al., 2009). Plant growth promoting rhizobacteria effect crop growth, nutrient uptake and yield of different agricultural crops (Parewa et al., 2014). Combined application of Rhizobium and Pseudomonas strains can improve the productivity of mung bean and nitrogen concentration in grains of mungbean (99\%), compared with the uninoculated control (Maqshoof et al. 2011; Jangra and Rajesh (2015) observed maximum growth in mungbean genotype MH-421 with dual inoculation of Rhizobium and PGPR. In pot experiment, it was reported that PGPR inoculation increased the growth of seedlings of Chickpea (Karnwal and Kumar, 2012) at $5 \%$ level of significance. The co-inoculation of PGPR and Rhizobium offers a novel prospective for future. Keeping in view the above discussion a pot study was conducted to evaluate the effect of co-inoculation on growth, nodulation, $\mathrm{N}$ content and yield in mungbean.

\section{MATERIALS AND METHODS}

Pot experiment was carried out in the experimental fields of Pulses Section, Department of Plant Breeding and Genetics, PAU, Ludhiana, during Kharif 2014 under glasshouse. The bacterial inoculums were prepared by inoculating them in respective broth 
followed by incubation at $40{ }^{\circ} \mathrm{C}$ for $48 \mathrm{~h}$ at $150 \mathrm{rpm}$. Medium black clayey soil from local Mungbean field was collected and autoclaved $3 \times\left(1 \mathrm{~h} 121^{\circ} \mathrm{C}\right)$ at $12 \mathrm{hrs}$ interval. One $\mathrm{Kg}$ sterilized soil was filled per pot. Seeds of Mungbean variety PAU 911 were procured from the Department of Plant Breeding and Genetics. Mungbean seeds of variety PAU 911 were inoculated with recommended Rhizobium strain and PGPR, as per treatments.

Mungbean seeds were surface sterilised with $0.1 \%$ sodium hypochlorite solution for $10 \mathrm{~min}$ and then washed three times with distilled water. Previously prepared $1 \mathrm{ml}$ samples of each inoculant will be uniformly applied on seeds as single and co-inoculation and ten seeds will be sown in each pot. Before inoculation, equal volume of different organisms (1:1) were mixed and allowed to stand for 30 minutes at room temperature. Inoculated seeds were dried at room temperature under shade before sowing. Crop was sown on $21^{\text {st }}$ July 2014 and harvested on October 14, 2014. The experiment was conducted in Randomized Block Design (RBD) with seven treatments (control,Rhizobium,Rhizobium+R-4,Rhizobium +R6,Rhizobium + S-5, Rhizobium + S-9 and Rhizobium + S-11) having five replications each.

Observations for number and dry weight of nodules, Fresh and dry weight of shoot and root, Plant height were recorded at 35 DAS and harvesting. Chlorophyll content and leghaemoglobin content were recorded at 35 DAS. Observations N-content in grains and straw, grain yield was recorded after harvesting. Three randomly selected plants were carefully uprooted from each pot at 35 DAS and harvesting, with root system intact. The roots were washed in running tap water and nodules were detached carefully with forceps and total number of nodules was counted. The number of nodules per plant was recorded by taking average. The detached nodules were dried in oven at $60^{\circ} \mathrm{C}$ for 2 days and their dry weight per plant was recorded in $\mathrm{mg}$.

Five randomly selected plants were uprooted from each pot and fresh weight of shoot and root per plant was measured in $\mathrm{gm}$. These were then dried in oven at $60^{\circ} \mathrm{C}$ for 2 days and dry weight per plant was measured in gm. Chlorophyll content was estimated by method of Witham et al 1971 and Leghaemoglobin content by method of Wilson and Reisenauer 1963. Grain yield from each pot (gm/pot) was recorded and the final grain yield was expressed in $\mathrm{gm} /$ plant. Nitrogen content in straw and seed was estimated by method of McKenzie and Wallace 1954 and expressed in $(\%)$.

\section{RESULTS AND DISCUSSION}

Rhizobium co-inoculated with different PGPR cultures (R-4, R-6, S-5,S-9 and S-11) showed increase in number of nodules (17.6, 24.3, 20.0, 11.6, and 22.3 number of nodules/plant) than Rhizobium alone (17.3 $\mathrm{nn} / \mathrm{plant}$ ) and control (11.3 nn/plant) (Fig.1). The increase in nodulation may be credited to their abilities to produce IAA, as its role is reported to regulate formation of nodules in legumes, due to suppression of pathogens (Mahmoud and Abd Alla, 2001) or nutrient mobilization (Ahemad and Khan, 2011). Application of different PGPR cultures i.e R-4, R-6, S-5, S-9 and S -11 along with Rhizobium further increased nodule dry weight ( $130-230 \mathrm{mg} / \mathrm{plant}$ ) as compared to Rhizobium alone (Table 1). Positive effect of inoculated Rhizobium on nodulation may be due to presence of either low or ineffective population of native rhizobia in soil (Chandra and Pareek, 2002).

At 35 days Rhizobium along with R-6 (10.1 gm/plant) showed maximum increase in shoot fresh weight as compared to Rhizobium along with R-4, S-5, S-9 and S -11 . Increase in fresh weight of shoots was observed in Rhizobium along with R-6 (13.7 gm/plant) and S-11 (11.44 gm/plant)at $5 \%$ level of significance during harvesting. A similar trend with root fresh weight was observed with co-inoculation of Rhizobium along with R-6 (0.65 and $1.70 \mathrm{~g} / \mathrm{plant})$ and S-11 (0.63 and 1.59 $\mathrm{gm} / \mathrm{plant}$ ) at $35 \mathrm{DAS}$ and harvesting (Table 1). Treatments with Rhizobium along with R-6 (2.3 gm/plant) showed increase in shoot dry weight as compared to Rhizobium (2.1 gm/plant) alone and control (1.8 g/ plant) at 35 DAS. Significant increase (at 5\%) was recorded in treatments with Rhizobium along with R-6 (4.01 gm/plant) and S-11 (3.79 gm/plant) at harvesting (Table 2). Dual inoculation of Rhizobium along with $\mathrm{R}$ -6 and S-11 showed higher root biomass at harvesting ( 0.80 and $0.77 \mathrm{gm} / \mathrm{plant}$ ) which was on par than at 35 DAS (0.22 and .2 gm/plant) (Table 2).

Highest Chlorophyll content was recorded by Rhizobium + R-6 (.28 mg/g fresh wt. of leaves) and Rhizobium $+\mathrm{S}-11$ (.23 mg/g fresh wt. of leaves). Mishra et al (2012) also reported that Rhizobium leguminosarumPR1 inoculation showed significant increase at $5 \%$ in $\mathrm{Chl} \mathrm{a}, \mathrm{Chl} \mathrm{b}$ and total $\mathrm{Chl}$ contents as compared to non -inoculated control plants in field pea. Maximum leghaemoglobin was recorded in Rhizobium with R-6 $(3.47 \mathrm{mg} / \mathrm{gm}$ of nodule), followed by S-11, S-9, R-4 and S-5 (3.01, 2.54, 1.43 and $1.29 \mathrm{mg} / \mathrm{gm}$ of nodule) repectively to ensure the proper fuction of root nodules.). Mishra et al. (2010) reported that co-inoculation of PGPR and Rhizobium resulted in maximum $45.9 \%$ increase in leghaemoglobin content as compared to Rhizobium inoculation alone in mungbean. Data regarding plant height revealed that Rhizobium inoculation and coinoculation significantly increased plant height over control, by $12 \%$. Maximum increase in plant height (23.7\%) over control and $10.8 \%$ over Rhizobium inoculation alone was recorded from coinoculation of Rhizobium + PGPR in mungbean under field conditions (Waseem et al 2004). Rhizobium coinoculated with R-4, R-6,S-5,S-9 and S-11 showed an increase in shoot length (38.8,42.4,39.4,38.4 and $40.4 \mathrm{~cm}$ ) respectively at 35 DAS. At harvesting Rhizo- 
bium inoculated along R-6 showed further increase in shoot length $(65 \mathrm{~cm})$ followed by S-11,S-9,R-4 and S$5(57,61,55$ and $55 \mathrm{~cm})$ respectively (Table 3$)$.

Rhizobium along with R-4, S-5,S-9 and S-11 application showed increased root length $(13.1,12.7,14.7$ and $17.5 \mathrm{~cm}$ ) respectively as compared to Rhizobium alone at 35 DAS. Maximum root length was recorded in co inoculation of Rhizobium along with R-6 $(31 \mathrm{~cm})$ at 75 DAS. Rhizobium inoculation alone and in combination with PGPR increases number of pods/ plant compared to uninoculated control at $5 \%$ level of significance. Rhizobium co-inoculated with R-4,R-6,S-5,S-9 and S11 also increased the number of pods per plant (6,10,7,8 and 13) as compared to control and Rhizobium alone. Significant increase $(5 \%)$ was observed in number of seeds per pod in Rhizobium co-inoculated with R-4, R-6, S-5, S-9 and S-11 (Table 4).

Nitrogen content is an important growth parameter, which has a direct bearing withbiological Nitrogen fixation . Data recorded on $\mathrm{N}$ content in mungbean

Table 1. Effect of co-inoculation of Rhizobium and PGPR on nodulation and plant fresh weight.

\begin{tabular}{lllllll}
\hline Treatments & $\begin{array}{l}\text { No. of nod- } \\
\text { ules/ plant }\end{array}$ & $\begin{array}{l}\text { Nodule dry } \\
\text { weight (mg/ } \\
\text { plant })\end{array}$ & $\begin{array}{l}\text { Shoot fresh weight (gm/ } \\
\text { plant) }\end{array}$ & $\begin{array}{l}\text { Root fresh weight (gm/ } \\
\text { plant) }\end{array}$ \\
\cline { 2 - 6 } & 35 DAS & 35 DAS & 35 DAS & Harvesting & 35 DAS & Harvesting \\
\hline Control & 11.3 & 90 & 4.81 & 6.20 & 0.36 & 1.0 \\
Rhizobium & 17.3 & 120 & 5.81 & 7.81 & 0.44 & 1.08 \\
Rhizobium + R-4 & 17.6 & 130 & 8.6 & 9.44 & 0.52 & 1.46 \\
Rhizobium + R-6 & 24.3 & 230 & 10.1 & 13.7 & 0.65 & 1.70 \\
Rhizobium + S-5 & 20.0 & 180 & 8.0 & 8.24 & 0.52 & 1.47 \\
Rhizobium + S-9 & 11.6 & 130 & 8.7 & 11.32 & 0.58 & 1.49 \\
Rhizobium + S-11 & 22.3 & 220 & 8.7 & 11.44 & 0.63 & 1.59 \\
CD@ 5\% & 1.2 & 7.6 & .81 & 0.60 & 0.68 & 0.61 \\
\hline
\end{tabular}

Table 2. Effect of co-inoculation of Rhizobium and PGPR on plant dry weight.

\begin{tabular}{lllcc}
\hline Treatments & \multicolumn{2}{l}{ Shoot dry weight (gm/plant) } & Root dry weight (gm/plant) \\
\cline { 2 - 5 } & 35 DAS & harvesting & 35 DAS & 75 DAS \\
\hline Control & 1.8 & 1.89 & 0.16 & 0.54 \\
Rhizobium & 2.1 & 2.47 & 0.19 & 0.58 \\
Rhizobium + R-4 & 2.1 & 3.04 & 0.18 & 0.60 \\
Rhizobium + R-6 & 2.3 & 4.01 & 0.22 & 0.80 \\
Rhizobium + S-5 & 1.5 & 2.07 & 0.12 & 0.69 \\
Rhizobium + S-9 & 2.0 & 3.62 & 0.2 & 0.70 \\
Rhizobium + S-11 & 2.5 & 3.79 & 0.2 & 0.77 \\
CD @ 5\% & 0.67 & 0.13 & 0.23 & 0.18 \\
\hline
\end{tabular}

Table 3. Effect of co-inoculation of Rhizobium and PGPR on chlorophyll, leghaemoglobin contents and plant height in mungbean.

\begin{tabular}{lllllll}
\hline Treatments & $\begin{array}{l}\text { Chlorophyll } \\
\text { content (mg/gm } \\
\text { fresh weight of } \\
\text { leaves) }\end{array}$ & $\begin{array}{l}\text { Leghaemoglobin } \\
\text { content (mg/gm } \\
\text { fresh weight of } \\
\text { nodules) }\end{array}$ & Shoot length (cm) & Root length (cm) \\
\cline { 2 - 7 } & 35 DAS & 35 DAS & 35 DAS & Harvesting & 35 DAS & harvesting \\
\hline Control & 0.08 & 0.79 & 35.4 & 52 & 11.3 & 24 \\
Rhizobium & 0.20 & 1.04 & 39.6 & 56 & 14.1 & 23 \\
Rhizobium + R-4 & 0.21 & 1.43 & 38.8 & 55 & 13.1 & 29 \\
Rhizobium + R-6 & 0.28 & 3.47 & 42.4 & 65 & 18.2 & 31 \\
Rhizobium + S-5 & 0.19 & 1.29 & 39.4 & 55 & 12.7 & 20 \\
Rhizobium + S-9 & 0.21 & 2.54 & 38.4 & 61 & 14.7 & 28 \\
Rhizobium + S-11 & 0.23 & 3.01 & 40.4 & 57 & 17.5 & 28.3 \\
CD@5\% & 0.12 & 0.90 & 0.18 & 0.67 & 0.18 & 0.76 \\
\hline
\end{tabular}

Table 4. Effect of dual-inoculation on yield attributing traits.

\begin{tabular}{llllll}
\hline Treatments & No. of pods /plant & No. of seeds per pod & Yield (gm/plant) & \multicolumn{2}{c}{ N content \% age } \\
\cline { 4 - 6 } & & & & Grains & Straw \\
\hline Control & 4 & 7 & 0.83 & 3.31 & 0.82 \\
Rhizobium & 5 & 8 & 1.44 & 3.48 & 1.03 \\
Rhizobium + R-4 & 6 & 9 & 1.32 & 3.53 & 1.26 \\
Rhizobium + R-6 & 10 & 12 & 1.70 & 4.02 & 2.32 \\
Rhizobium + S-5 & 7 & 9 & 1.23 & 3.55 & 1.17 \\
Rhizobium + S-9 & 8 & 10 & 1.44 & 3.62 & 1.36 \\
Rhizobium + S-11 & 13 & 10 & 1.60 & 3.75 & 1.39 \\
CD @5\% & 0.30 & 0.18 & 0.15 & 0.62 & 0.76 \\
\hline
\end{tabular}


grains in Table 4 depicts that all the treatments increased the $\mathrm{N}$ content over control which is beneficial in increasing the level of protein content. $\mathrm{N}$ content ranged from 3.31 to $4.02 \%$ in grains and from 0.82 to 2.32 in straw. Treatments of R-6 and S-11 alongwith Rhizobium showed an increase in $\mathrm{N}$ content in grains and straw as compared to Rhizobium Grain yield is the most important economic trait. The grain yield was recorded at harvesting stage to analyse best cultures that can be further used as biofertilizers in mungbean. Inoculation of Rhizobium and PGPR considerably enhanced the grain yield, while the effect was more pronounced when they are applied in combination as compared to un-inoculated ones. The highest grain yield was produced by R-6 along with Rhizobium (1.70 gm/ plant) followed by Rhizobium alone (1.44 gm/plant). Combination of Bradyrhizobium sp. MN-S + B. subtilis M6 remarkably improved grain yield to $1478 \mathrm{~kg} /$ ha, which was $22 \%$ higher than the Bradyrhizobium sp. MN-S alone (1216 kg/ ha) and 44\% higher than the un-inoculated control (947 $\mathrm{kg} / \mathrm{ha})$ in mungbean (Mohsin et al., 2012). Bansal (2009) reported that combined inoculation of mung bean seeds with Rhizobium + PGPR + PSB gave significantly higher (at $5 \%$ ) grain yield as compared to Rhizobium alone

\section{Conclusion}

During the present study native PGPR isolates R-6 and S-11 along with recommended Rhizobium improved plant growth, symbiotic parameters and grain yield over Rhizobium alone. The use of PGPR as inoculants is an efficient approach to replace chemical fertilizers ,pesticides which have numerous side effects to sustainable agriculture. Further these rhizobacterial strains can be used as biofertilizer for a wide variety of legume

\section{REFERENCES}

Ahemad, M. and Khan, M.S. (2011) Pseudomonas aeruginosa strain PS1 enhances growth parameters of green gram (Vigna radiata L.Wilczek) in insecticide-stressed soils. J Pest Sci., 84:123-31.

Anjum, M.S., Ahmed, Z.I. and Rauf, C.A. (2006). Effect of Rhizobium Inoculation and Nitrogen Fertilizer on Yield and Yield Components of Mungbean. Int. J. Agr. Biol., 8: $238-40$

Anonymous (2013). Package and practice for kharif crops. Pp 59. Punjab Agricultural University, Ludhiana.

Bansal, R.K. (2009). Synergistic effect of Rhizobium, PSB and PGPR on nodulation and grain yield of mungbean. J. Food legume, 22:37-39.

Burchi, F., Fanzo, J. and Frison, E. (2011) The role of food and nutrition system approaches in tackling $\mathrm{h} i \mathrm{~d} d \mathrm{e} \mathrm{n}$ hunger. Int. J. Environ Res Public Health, 8:358-73

Chandra, R. and Pareek, R.P. (2002). Effect of Rhizobacteria in urdbean and lentil. Indian J. Pulses Res., 15: 152-55

Fuzzaman, A.M., Hossen, F.A., Ismail, M.R., Hoque, M.A., Islam,M.Z., Shahidullah, S.M. and Meon, S. (2009) Effi- ciency of plant growth promoting Rhizobacteria (PGPR) for the enhancement of rice growth. Afr. $J$. Biotechnol., 8: 1247-52.

Jangra, D. and Rajesh, Y. (2015) Genotypic variation for response to Rhizobium spp. and Piriformospora indica for yield and yield components in mungbean (Vigna radiata L. Wilczek). International Journal of Applied Biology and Pharmaceutical Technology, 6: 26772

Karnwal, A. and Kumar, V. (2012) Influence of plant growth promoting rhizobacteria (PGPR) on the growth of chickpea (Cicer arietinum). Ann. Food Sci Technol., 1: $1-6$

Khanna, V. and Sharma, P. (2011) Potential for enhancing lentil (Lens culinaris) productivity by co-inoculation with PSB, plant growth promoting rhizobacteria and Rhizobium. Ind. J. Agril. Sci., 81: 932- 34

Mahmoud, A.L.E and Abd- Alla, M.H (2001). Siderophore production by some microorganisms and their effect on Bradybrhizobium - mungbean Symbiosis. Int. J. Agric. Biol., 3:157-62.

Maqshoof, A., Muhammad, A., Naeem, A. and Zahir, A. (2011). The combined application of rhizobial strains and plant growth promoting rhizobacteria improves growth and productivity of mung bean (Vigna radiata L.) under salt-stressed conditions. Annals. Microbiol., 62: 1321-30.

McKenzie, H.A. and Wallace, H.S. (1954). The Kjeldahl determination of nitrogen: a critical study of digestion conditions-temperature, catalyst and oxidising agent. Aust. J. Chem., 7: 55.

Mishra, P.K., Bisht, S.C., Ruwari, P., Joshi, G.K., Singh, G., Bisht, J.K. and Bhatt, J.C. (2010). Bioassociative effect of cold tolerant Pseudomonas spp. And Rhizobium leguminosarum- PR1 on iron acquisition ,nutrient uptake and growth of lentil (Lens culinaris L.). Europ. J. Soil Biol., 47:35-43

Mishra, P.K.., Bisht, S.C., Bisht, J.K., and Bhatt, J.C. (2012). Cold-tolerant PGPRs as bioinoculants for stress management. Bacteria in Agrobiology, 95:118-20.

Mia, M.A., Shamsuddin, Z.H. and Mahmood, M. (2010). Effects of rhizobia and plant growth promoting bacteria inoculation on germination and seedling vigor of lowland rice. Afr. J. Biotechnol., 11: 3758-65.

Mohsin, T., Sohail, H., Tahira, Y. and Amanat, A. (2012). Non-rhizobial bacteria for improved nodulation and grain yield of mung bean (Vigna radiata (L.) Wilczek). Afr. J. Biotechnol., 11: 15012-19.

Parewa, H.P., Yadav, J., Rakshit, A., Meena, V.S., and Karthikeyan, N. (2014) Plant growth promoting rhizobacteria enhance growth and nutrient uptake of crops. Agriculture for Sustainable Development, 2:101116.

Waseem, R., Javed, A., Muhammad, A. and Sohail, Y. (2004). Growth nodulation and yield of mungbean (Vigna radiata $\mathrm{L}$ ) as influenced by coinoculation with Rhizobium and Plant growth promoting rhizobacteria. Pak. J. Agri. Sci., 41:3-4.

Wilson, D.O. and Reisenauer, H.M. (1963). Determination of leghaemoglobin in legume nodules. Anal. Biochem., 6: $27-$ 30.

Witham, F.H., Bladydes, D.F. and Delvins, R.M. (1971) Experiments in plant physiology. Pp 245. Van Nostrand Reinhold, New York. 